\title{
Improved Design Scenario for Composite Airframe Structures
}

\author{
Richard Degenhardt ${ }^{1}$ Jan Tessmer ${ }^{2}$ \\ DLR, Institute of Composite Structures and Adaptive Systems, 38108 Braunschweig, Germany
}

\begin{abstract}
European aircraft industry demands for reduced development and operating costs, by $20 \%$ and $50 \%$ in the short and long term, respectively. Contributions to this aim are provided by the completed project POSICOSS and the running follow-up project COCOMAT, both supported by the European Commission. As an important contribution to cost reduction a decrease in structural weight can be reached by exploiting considerable reserves in primary fibre composite fuselage structures through an accurate and reliable simulation of postbuckling and collapse. The POSICOSS team developed fast procedures for postbuckling analysis of fibre composite stiffened panels, created comprehensive experimental data bases and derived design guidelines. COCOMAT builds up on the POSICOSS results and considers in addition the simulation of collapse by taking degradation into account. The main objective is a future design scenario for composite stiffened panels which allows the exploiting of considerable reserves in primary fibre composite fuselage structures. The results comprise an extended experimental data base, degradation models, improved certification and design tools as well as design guidelines. The paper deals with the main objectives of the project COCOMAT, the general status of the progress as well as DLR's first results.
\end{abstract}

\section{Introduction}

$\mathrm{E}$ EUROPEAN aircraft industry demands for reduced development and operating costs, by $20 \%$ and $50 \%$ in the short and long term, respectively. Supported by the European Commission the 4-year project COCOMAT, which started in January 2004, contributes to this aim. COCOMAT stands for Improved MATerial Exploitation at Safe Design of COmposite Airframe Structures by Accurate Simulation of Collapse. The project is co-ordinated by DLR, Institute of Composite Structures and Adaptive Systems. It aims at allowing for a structural weight reduction by exploiting considerable reserves in primary fibre composite fuselage structures through an accurate and reliable simulation of postbuckling and collapse (cf. [4] and [11]).

The COCOMAT project is fully based upon the results of the POSICOSS project, which lasted from January 2000 to September 2004. POSICOSS is the acronym of Improved POstbuckling SImulation for Design of Fibre COmposite Stiffened Fuselage Structures. The POSICOSS team has developed improved, fast and reliable procedures for buckling and postbuckling analysis of fibre composite stiffened panels of future fuselage structures. For the purpose of validation comprehensive experimental data bases were created. Finally, design guidelines were derived. An overview about the POSICOSS project can be found in [1]. Some of DLR's results are published in [2] and [3].

The COCOMAT project extends the POSICOSS results and goes beyond by a simulation of collapse. That requires knowing about degradation due to static as well as low cycle loading in the postbuckling range. It is wellknown that thin-walled structures made of carbon fibre reinforced plastics are able to tolerate repeated buckling without any change in their buckling behaviour. However, it has to be found out, how deep into the postbuckling regime the loading can be extended without severely damaging the structure, and how the behaviour can be predicted by fast and precise simulation procedures. This issue is dealt with by COCOMAT.

COCOMAT will improve existing slow and fast simulation tools and will set up design guidelines for stiffened panels which take skin stringer separation and material degradation into account. Reliable fast tools allow for an economic design process, whereas very accurate but necessarily slow tools are required for the final

\footnotetext{
${ }^{1}$ Group leader, Department Structural Mechanics, Lilienthalplatz 7, 38116 Braunschweig, Germany

${ }^{2}$ Head of department, Department Structural Mechanics, Lilienthalplatz 7, 38116 Braunschweig, Germany
} 
certification. The results will comprise a substantially extended data base on material properties and on collapse of undamaged and pre-damaged structures subjected to static and low cycle loading, degradation models, improved slow and fast computation tools as well as design guidelines.

Regarding loads and characteristic dimensions, the projects POSICOSS and COCOMAT are oriented towards an application in the field of fuselage structures, but the results are transferable to other airframe structures as well. With the new design guidelines the aircraft industry will have a tool at its disposal, which substantially contributes to the objectives of reducing development and operating costs.

\section{Improved design scenario for composite airframe panels - Objective of COCOMAT}

COCOMAT mainly strives for accomplishing the large step from the current to a future design scenario of typical stringer stiffened composite panels demonstrated in Figure 1. The left graph illustrates a simplified loadshortening curve and highlights the current industrial design scenario. Three different regions can be specified. Region I covers loads allowed under operating flight conditions and is bounded by Limit Load (LL); region II is the safety region and extends up to Ultimate Load (UL); region III comprises the not allowed area which reaches up to Collapse. In aircraft design Ultimate Load amounts to $150 \%$ of Limit Load. There is still a large unexploited structural reserve capacity between the current Ultimate Load and Collapse. The right graph of Figure 1 depicts the design scenario aspired in future, where Ultimate Load is shifted towards Collapse as close as possible. Through that move the onset of degradation appears no longer in the not allowed region III but already in the safety region II. This is comparable to metallic structures where plasticity is permitted in the safety region. However, it must be guaranteed that in any case the onset of degradation must not occur below Limit Load. Moreover, the extension requires an accurate and reliable simulation of Collapse, which means to take into account degradation under static as well as under low cycle loading to assure its limited progression.

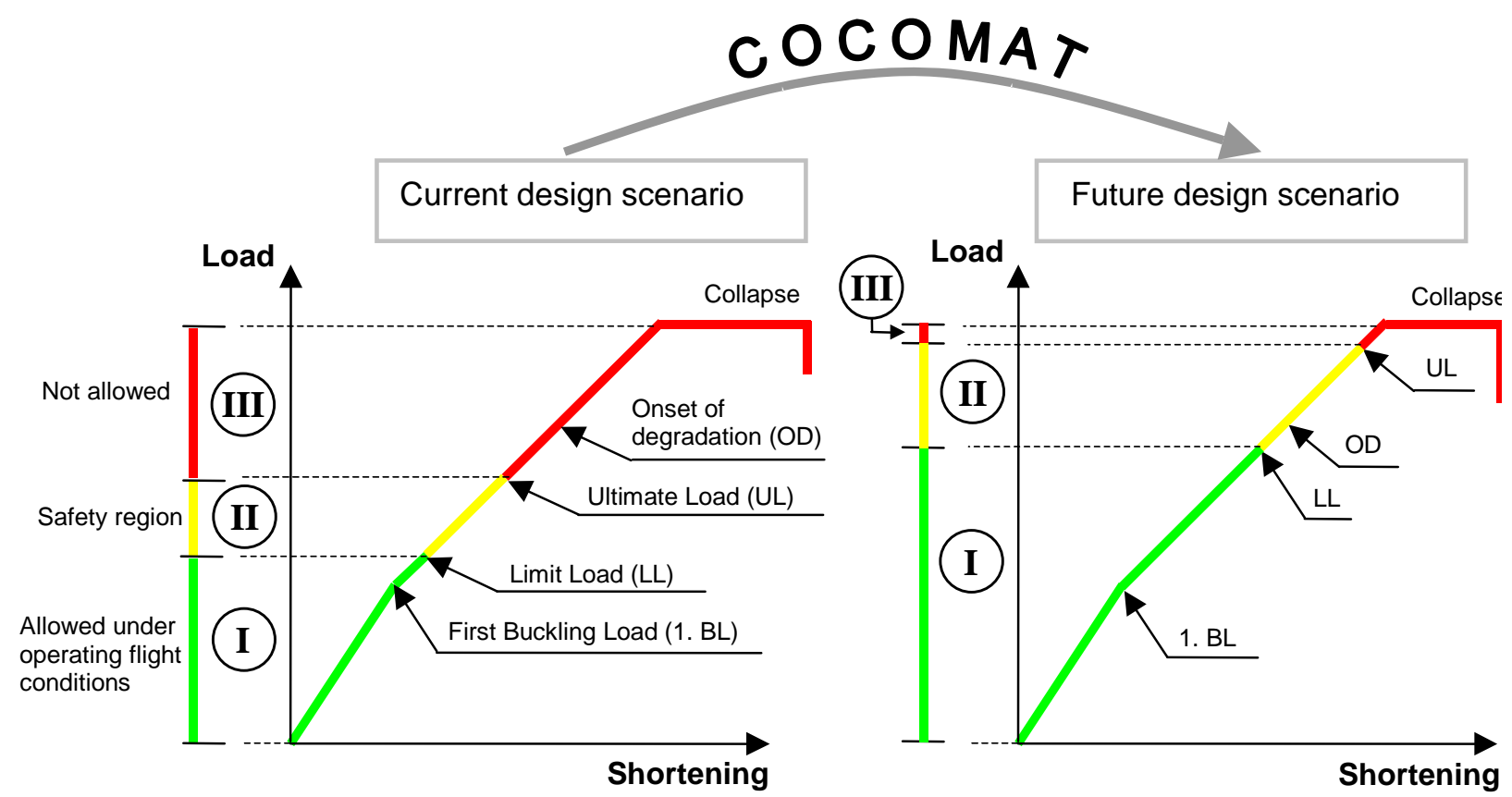

Figure 1. Current and future design scenarios for typical stringer stiffened composite panels [4].

\section{Consortium}

The COCOMAT consortium merges knowledge from 5 large industrial partners (AGUSTA from Italy, GAMESA from Spain, HAI from Greece, IAI from Israel and PZL from Poland), 2 Small and Medium Enterprises (SAMTECH from Belgium and SMR from Switzerland), 3 research establishments (DLR from Germany, FOI from Sweden and CRC-ACS from Australia) and 5 universities (Politecnico di Milano from Italy, RWTH Aachen and 
University of Karlsruhe from Germany, TECHNION from Israel and Technical University of Riga from Latvia). The work builds up on the knowledge, the experience, the results and especially the fast tools of the 5th Framework Project POSICOSS.

\section{Workpackages}

The partners co-operate in the following six technical work packages:

- WP 1: Benchmarking on collapse analysis of undamaged and damaged panels with existing tools: Knowledge of the partners is compared and the deficiencies of existing software are identified.

- WP 2: Material characterisation, degradation investigation and design of panels for static and cyclic tests: Material properties are characterized, degradation models are developed and test panels are designed as to the requirements of research in order to overcome the deficiencies.

- WP 3: Development of improved simulation procedures for collapse: Slow certification tools and fast design tools are developed and validated by the tests.

- WP 4: Manufacture, inspection and testing by static and cyclic loading of undamaged panels: The experimental data base is extended by testing of undamaged panels.

- WP 5: Manufacture, inspection and testing by static and cyclic loading of pre-damaged panels: The experimental data base is extended by testing of pre-damaged panels.

- WP 6: Design guidelines and industrial validation: All project results are assembled and final design guidelines are derived. The tools are validated by the industrial partners.

Industrial partners bring in their experience with design and manufacture of real shells; research partners contribute knowledge on testing and on development of simulation tools. Design guidelines are defined in common, and the developed tools are validated by the industrial partners.

\section{Expected results and benefits}

The project results will comprise a substantially extended data base on material properties and on collapse of undamaged and pre-damaged statically and cyclically loaded structures, degradation models, improved slow and fast computation tools for statically loaded structures as well as design guidelines, which take skin stringer separation and material degradation into account. The experimental data base is indispensable for validation of the analytically developed degradation models, which will be implemented into the new tools, and for verification of the computed results as well. Reliable fast tools will allow for an economic design process, whereas very accurate but necessarily slow tools are required for the final certification.

Although, with respect to loads and characteristic dimensions, this project is oriented towards an application in the fields of fuselage structures, the results will be transferable to other airframe structures as well. With the new design guidelines the European aircraft industry will have a tool at its disposal, which substantially contributes to the objectives of reducing development and operating costs. That provides the chance for decisive improvements in competitiveness of future European aircraft.

The traditional aircraft design can be replaced by an advanced procedure including degradation models for composite structures. One main benefit of the application of the new tools and design guidelines will be a considerably reduced structural weight at safety not impaired. In addition, the developed tools also reduce the design and analysis time by one order of magnitude and thus, they substantially improve the response-to-market time of industrial developments.

\section{Selected results}

During the first three project years the COCOMAT partners worked mainly on the first five technical workpackages. This section gives a general summary of the status of each workpackage and presents some of DLR's first results. Because workpackage 5 is in a preliminary stage this paper concentrates on the first 4 workpackages. A list of papers published by the partners so far can be found at www.cocomat.de.

\section{A. Workpackage 1}

The partners selected two panel tests from the POSICOSS projects as benchmarks on undamaged structures. In order to obtain test results of a comparable pre-damaged panel one panel from the POSICOSS project was refurbished, a minor damage was fixed, then it was pre-damaged by IAI and tested by TECHNION. In addition, the 
consortium exchanged test results of pre-damaged benchmark structures with Airbus Germany. The partners applied different finite element tools on the benchmarks in order to simulate the structural behaviour up to collapse. They identified abilities and deficiencies of the simulation of degradation.

Some detailed results on the two undamaged benchmarks are published in [9]. As an example Figure 2 shows the load shortening curve of one undamaged benchmark (axially loaded CFRP panel) provided by DLR and the comparison with simulations by means of the commercial tools ABAQUS and NASTRAN. There is a good agreement of all curves from the prebuckling region up to the first global buckling (at $1.4 \mathrm{~mm}$ shortening) where the stringers buckle. From that point there is still a good agreement between the different numerical simulations which take imperfections into account. The agreement with the experiment becomes worse. However, in that deep postbuckling region the simulation is not expected to agree with the test because degradation (e.g. material degradation, skin-stringer separation or the delamination in the stiffener blade) is not

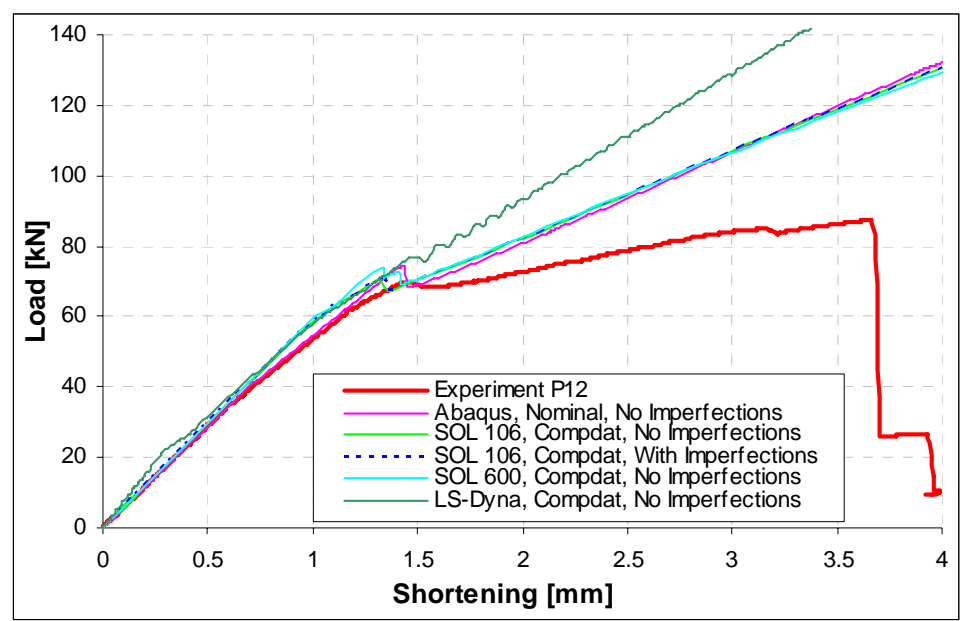

Figure 2. Some results from WP 1 [6].

taken into account. In the frame of WP3 the simulation tools will be improved in that way that the effect of most important types of degradation can be simulated. In addition, the modeling of the lateral boundary conditions largely influences the results in that load region. More details can be found in [8].

\section{B. Workpackage 2}

The partners involved in Task 2.1 characterised the material properties of the specimens with and without damages, manufactured of the material IM7/8552 UD, 985-GT6-135 and IM7/8552. DLR has determined the material properties of specimens, according to the German standard. The specimens are manufactured of IM7/8552 UD by AERNNOVA. Additionally, so called small specimen tests, according to compression after impact tests (CAI) have been conducted to examine a possible stiffness reduction as a function of delamination size. Fig. 3 illustrates the DLR's test set-up for the CAI tests and one of the specimens with applied strain gauges. All DLR's results are summarized in [10].

The objectives of Task 2.2 are improved degradation models which are
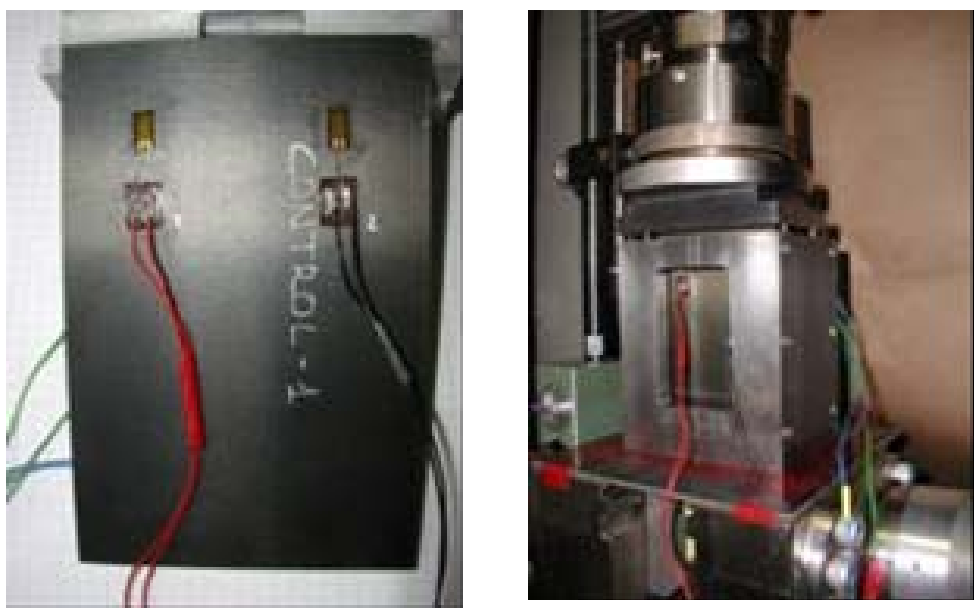

Figure 3. DLR's test set-up for CAI tests and specimens with strain gauges [9] .

needed for the slow certification tools and fast design tools in WP 3. These will be obtained by test results. Planned specimens for the investigation of degradation are plates or small structures made a skin with one or two stiffeners. Partners involved in the experimental activity are providing tables and graphs to be used for the development of new procedures or the improvement of existing slow and fast numerical codes in order to consider combined effects of damages and compressive loading. The test campaign is almost finished. Efforts are spent on a critical review of the collected data and on an improvement of the tests in order to characterize degradation onset. Some of the partners also performed numerical simulations of the specimen behaviour in order to better understand degradation mechanisms. 
Task 2.3 has as objective the design and analysis of stiffened panels which shall be manufactured and tested in WP4. The group designed two kinds of panels: validation panels and industrial panels. The validation panels are designed as to specific limiting aspects of application of the software to be verified, e.g. type of shell theory, type of buckling before postbuckling, mild or strong stiffness reduction in the postbuckling regime, multiple or single modes of buckling before postbuckling. These panels should have a significant postbuckling range up to collapse and an early onset of degradation. The industrial panels are designed in regard to industrial applications, mainly by existing procedures used in day-to-day industrial design practice. In total the COCOMAT consortium designed 85 panels 6 of them were selected for manufacturing and testing. The first validation panel (Design

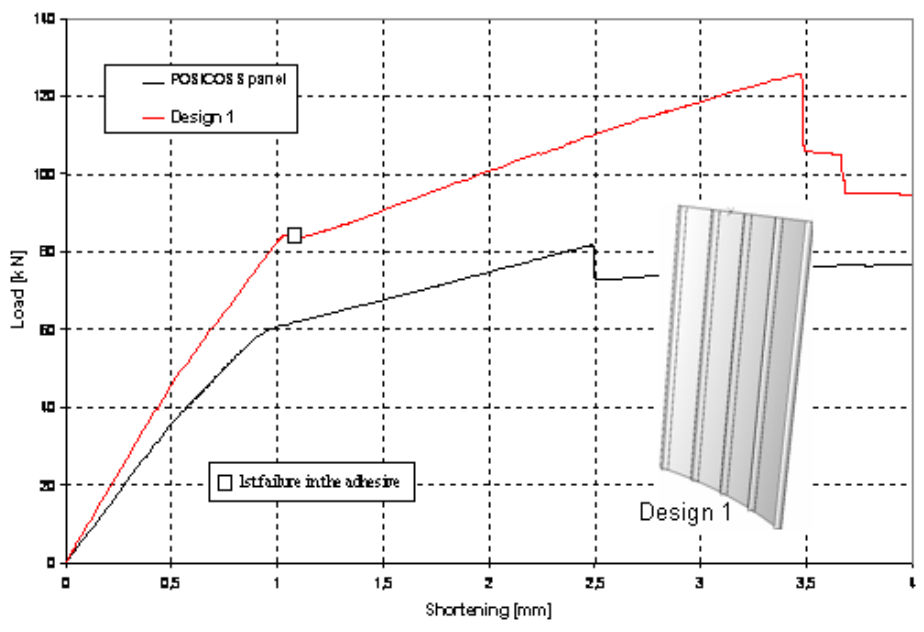

Figure 4. COCOMAT panel design in comparison to the start design from POSICOSS [8]. 1) was designed in co-operation with CRC-ACS, HAI and DLR. The initial configuration for the design process was taken from the POSICOSS project. The objective was to increase further the postbuckling region, especially to have a certain load capacity after the first global buckling. The reason is that the influence of skin-stringer separation on the collapse load should be investigated and this kind of degradation usually occurs after the first global stringer buckling. Several parametric studies for the variation of the lay-up of the skin and stringer, number stringers, stringer geometry and position of the stringers were performed. During the design process the onset of different kinds of degradation, as skin-stringer separation, delamination in the stringer blade and failure in the composite laminate structure have been estimated by simple extension of the available software tool. In order to check the influence of degradation on collapse the panels with a large postbuckling region and the indication of skin-stringer separation (failure in the adhesive layer) as early failure mode were favoured. There was also a second important change of Design 1 in comparison to the POSICOSS one. For Design 1 the clamping boundary conditions of the lateral edges of the panel, which were applied to all POSICOSS experiments, were released because the modelling of these boundary conditions showed a significant influence on the axial stiffness in the postbuckling region after the first global stringer buckling (cf. Figure 2). However, in order to avoid an early start of skin buckling due to the free lateral edges the stringers were moved in circumferential direction to support these edges. In addition, computations on different designs were performed in order to ensure that the onset of skin-stringer separation starts in the middle stringers and not in the outer ones. Figure 4 illustrates the load-shortening curve of this design in comparison to a POSICOSS design. For the Design 1 there is a large postbuckling region, even after the first global stringer buckling and the stringer buckling starts in the middle of the panel. DLR's experience in the designing of panels within the projects POSICOSS and COCOMAT is explained in more detail in [6].

\section{Workpackage 3}

In WP3 slow and fast computational tools, which take degradation into account, are developed and improved. Very accurate but necessarily slow tools are required for the final certification, whereas reliable fast tools reducing design and analysis time by an order of magnitude, will allow for an economic design process. Finally, all tools are validated by means of the experimental results obtained from the other workpackages.

Task 3.1 concentrates on the improvement of slow certification simulation tools. Figure 5 illustrates the whole family of slow computational tools including the degradation models considered in that task.

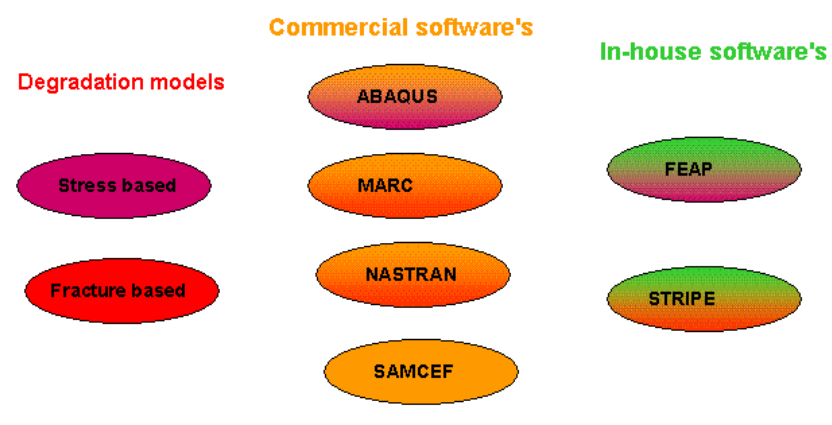

Stress based failure criteria: Tsai Hill, Maximum stress, Minimum strain, Puck Fracture based failure criteria: $V C C T$ energy release rates

Figure 5. Family of slow certification computational tools within Task 3.1. 
For simulating the skin-stringer separation of composite structures DLR developed a ABAQUS user subroutine and applied it to calculate the structural behaviour of Design 1 up to collapse. This panel was tested in WP 4 and a comparison, which shows good agreement, is given in Section D (cf. Figure 8). However, concerning the buckling shapes there are differences which could not be clarified. Detailed results can be found in [9].

Task 3.2 concentrates on the improvement of design procedures for the fast simulation of collapse behaviour of stringer stiffened fibre composite panels. The tools will be faster at least by a factor of 10 than respective Finite Element (FE) simulations at an accuracy, which is sufficient for design purposes. A graphical model representing overall precision and computing time of fast simulation procedures with degradation between all partners involved in that task is presented in Figure 7.

DLR is considering iBUCK which was developed in a previous project. iBUCK is a

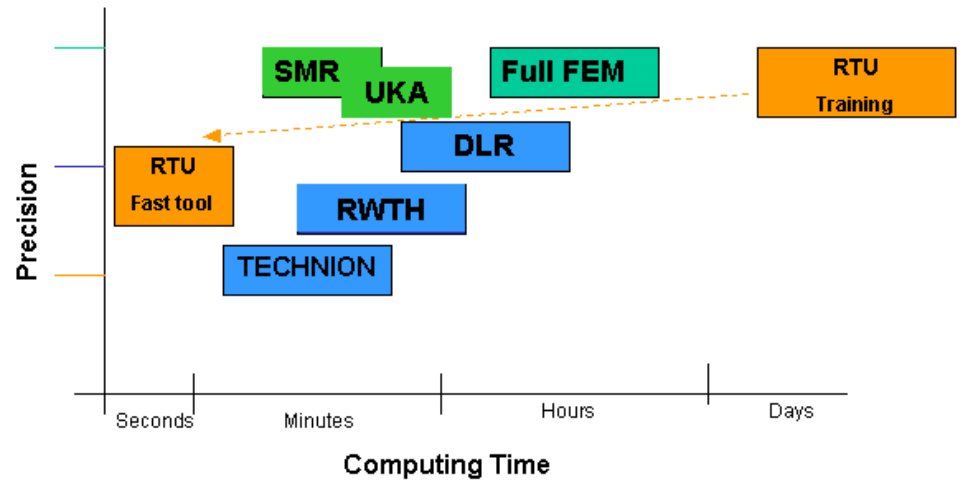

Figure 6. Family of fast design computational tools within Task 3.1.

tool for the fast simulation of the postbuckling behaviour of aerospace structures [5]. The model is based on the Donnell type shell equations for thin, slightly curved shells that undergo large deflections. Stringers are considered as structural elements with independent degrees of freedom and are not "smeared" onto the skin. Continuity in terms of rotation at the interface skin/stiffeners and in terms of end-shortening is enforced. Local and global buckling modes are superposed, where local buckling is defined as skin buckling and skin-induced stiffener rotation within a bay. During local buckling, the stiffeners themselves are not allowed to deflect in out-of-plane direction. During global buckling, that is, buckling across several bays, the stringers may deflect in out-of-plane direction, whereas the frames, being much heavier than the stringers, are fixed in out-of-plane direction. The panel may be loaded axially and bi-axially. In addition, one load case that is of special interest for the aircraft industry is included: the loading by an external bending moment in circumferential direction which may act in an opening or closing mode. All external loads may be applied individually or in combination. In COCOMAT skin-stringer separation is implemented into iBUCK. Figure 8 illustrates one postbuckling shape obtained by iBUCK.

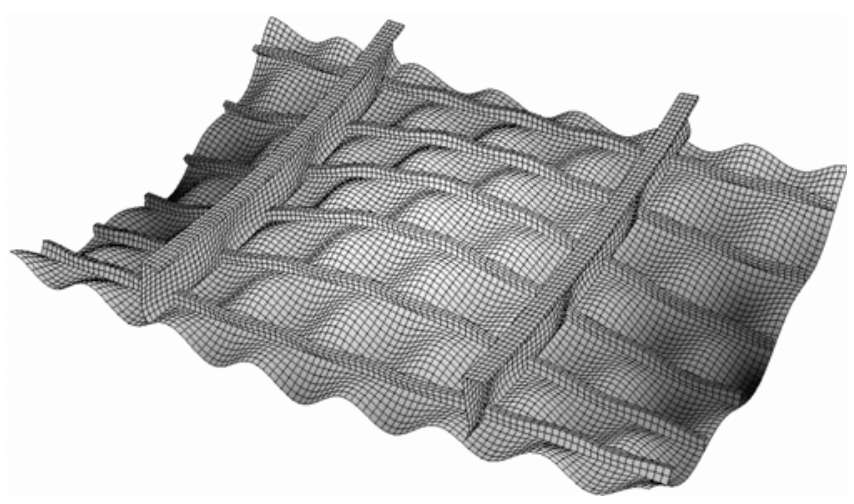

Figure 7. Postbuckling shape obtained by iBUCK [5].

\section{Workpackage 4 and 5}

The main objective of WP 4 is the extension of the experimental data base on collapse of undamaged stringer stiffened composite panels under static and low cycle loading. Task 4.1 concentrates on the manufacturing and nondestructive inspection of undamaged stiffened curved panels. The partners are munacturing 6 different panel types designed in Task 2.3. In Task 4.3 the panels are loaded cyclically by several thousands cycles until a certain level (e.g. 70-95\% of the expected collapse load) and finally until collapse. Cyclic loading is understood in that project as repeated static loading. In WP 5 nominally nominally the same panels are considered for manuacturing and testing by static and cyclic loading. The difference to WP 4 is that these panels will be pre-damaged by impact or a teflon layer between skin and stringer. Figure 8 gives an overview about all panel tests planned in WP 4 and WP 5 .

Figure 9 shows as an example first collapse test results obtained by DLR and the comparison with the simulation from WP 3. More details are given in [7]. 


\begin{tabular}{|c|c|c|c|c|c|c|c|}
\hline \multicolumn{8}{|c|}{ Number and kind of panel tests } \\
\hline \multirow{3}{*}{ Tasks } & \multicolumn{2}{|c|}{ GAMESA - DLR } & \multirow{2}{*}{$\begin{array}{c}\text { PZL } \\
\text { Design } 3\end{array}$} & \multirow{2}{*}{$\begin{array}{c}\begin{array}{c}\text { AGUSTA - } \\
\text { POLIMI }\end{array} \\
\text { Design } 5\end{array}$} & \multicolumn{2}{|c|}{ IAI - TECHNION } & \multirow[b]{3}{*}{$\sum_{\bar{D}}$} \\
\hline & Design 1 & Design 2 & & & Design 4 & Design 6 & \\
\hline & \multicolumn{2}{|c|}{ Compression } & Compression & $\begin{array}{l}\text { Combined } \\
\text { shear+ } \\
\text { compression }\end{array}$ & $\begin{array}{c}\text { Compres } \\
\text { sion }\end{array}$ & Shear & \\
\hline \multicolumn{8}{|c|}{ WP 4: Manufacture, inspection and testing by static and cyclic loading of undamaged panels from WP2 } \\
\hline Task 4.2: Buckling tests until collapse by static loading & 2 & 1 & $1 *$ & $2 *$ & - & - & 6 \\
\hline Task 4.3: Cyclic buckling tests & 2 & 1 & 2 * & 2 * & 2 & 4 * & 13 \\
\hline \multicolumn{8}{|c|}{ WP 5: Manufacture, inspection and testing by static and cyclic loading of pre-damaged panels from WP2 } \\
\hline Task 5.3: Buckling tests until collapse by static loading & 2 & 1 & $1 *$ & 2 * & 1 & 2 * & 9 \\
\hline Task 5.4: Cyclic buckling tests & 2 & 1 & \begin{tabular}{l|l|}
$2 *$ \\
\end{tabular} & 4 * & 2 & 4 * & 15 \\
\hline \multirow{2}{*}{ Sum } & 8 & 4 & \begin{tabular}{l|l}
6 & \\
\end{tabular} & 10 & 5 & 10 & \multirow{2}{*}{43} \\
\hline & \multicolumn{2}{|c|}{12} & 6 & 10 & \multicolumn{2}{|c|}{15} & \\
\hline
\end{tabular}

Figure 8. Overview and kind of panel tests.

\section{Summary}

The main objective of the running COCOMAT project is the future design scenario for stringer stiffened CFRP panels (cf. Fig. 1). COCOMAT builds up on the results of the finished POSICOSS project and considers in addition simulation of collapse by taking degradation into account. The results comprise an extended experimental data base, degradation models, improved certification and design tools as well as design guidelines. This paper deals with the main objectives and expected results of the project COCOMAT as well as DLR's first results.

\section{Acknowledgments}

The project COCOMAT is supported by the European Commission, Priority Aeronautics and Space, Contract AST3-CT-

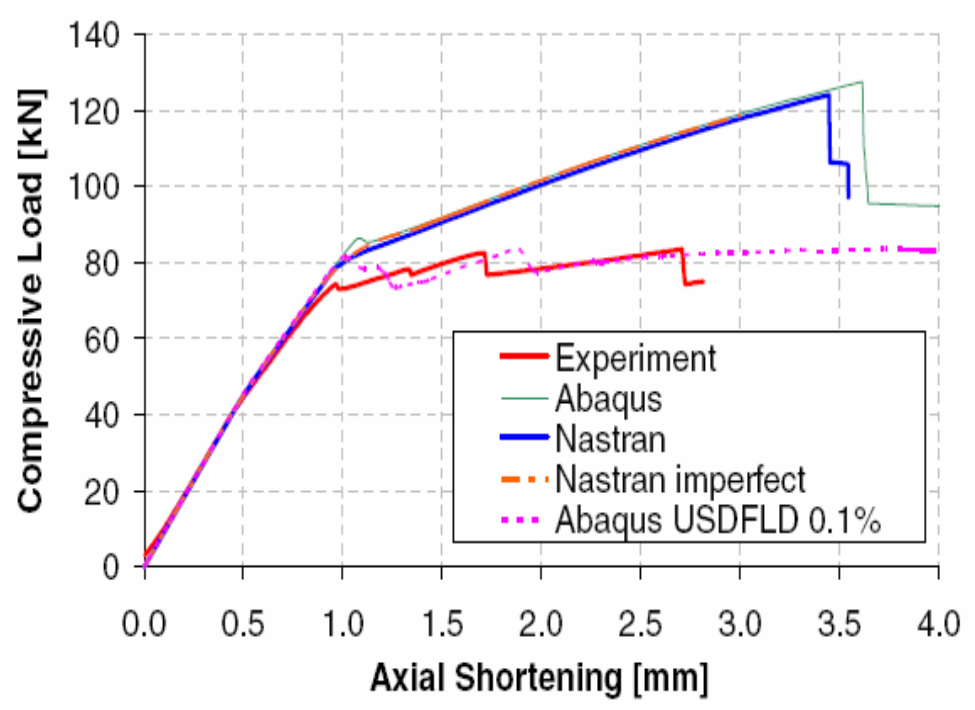
2003-502723. All support is gratefully acknowledged.

\section{References}

Periodicals

${ }^{1}$ Zimmermann R., Rolfes R., "POSICOSS - Improved postbuckling simulation for design of fibre composite stiffened fuselage structures”, Composite Structures, Vol. 73 (2006), pp. 171-174.

${ }^{2}$ Kling A., Degenhardt R., Zimmermann R., “A hybrid subspace analysis procedure for non-linear postbuckling calculation”, Composite Structures, Vol. 73 (2006), pp. 171-174.

${ }^{3}$ Zimmermann R., Klein H., Kling A., "Buckling and Postbuckling analysis of stringer stiffened fibre composite curved panels”, Composite Structures, Vol. 73 (2006), pp. 150-161

${ }^{4}$ Degenhardt R., Rolfes R., Zimmermann R., Rohwer K., "COCOMAT - Improved MATerial exploitation at safe design of COmposite airframe structures by accurate simulation of COllapse”, Composite Structures, Vol. 73 (2006), pp. 175-17.

${ }^{5}$ Bürmann P., Rolfes R., Tessmer J., Schagerl M., “A semi-analytical model for local post-buckling analysis of stringer- and frame-stiffened cylindrical panels”, Thin-Walled Structures, Vol. 44 (2006), pp. 102-114.

${ }^{6}$ Degenhardt R., Kling A., Rohwer K., "Design and Analysis of Stiffened Composite Panels Including Postbuckling and Collapse”, Journal of Computers and Structures, (paper accepted) 
${ }^{7}$ Orifici A. C., Thomson R. S., Degenhardt R., Kling A., Rohwer K., Bayandor J, "Degradation investigation in a postbuckling composite stiffened fuselage panel”, Journal of Composite Structures, doi: 10.1016/j.compstruct.2007.01.012.

${ }^{8}$ Degenhardt R., Kling A., Klein H., Hillger W., Goetting Ch., Zimmermann R., Rohwer K., Gleiter A., "Experiments on Buckling and Postbuckling of Thin-Walled CFRP Structures using Advanced Measurement Systems”, International Journal of Structural Stability and Dynamics, (paper accepted, to be printed in June 2007, Vol. 7, n 2)

\section{Proceedings}

${ }^{9}$ Orifici, A. C., Thomson, R. S., Gunnion, A. J., Degenhardt, R., Abramovich, H., Bayandor, J., "Benchmark finite element simulations of postbuckling composite stiffened panels”, 11th Australian International Aerospace Congress, Melbourne, Australia, 13-17 March, 2005.

Reports, Theses, and Individual Papers

${ }^{10}$ Kling A., Degenhardt R., "Characterization of material properties - Results of the EU project COCOMAT”, Internal DLR report, IB 131-2006, April 2006.

Private Communications and Web Sites

${ }^{11}$ www.cocomat.de 